\title{
On Analog Computation of Vector-Valued Functions in Clustered Wireless Sensor Networks
}

\author{
Mario Goldenbaum ${ }^{\star \ddagger}$, Holger Boche and Sławomir Stańczak $^{\star \dagger}$ \\ * Lehrstuhl für Informationstheorie und theoretische Informationstechnik, \\ Technische Universität Berlin, Einsteinufer 25, 10587 Berlin, Germany \\ $\ddagger$ Lehrstuhl für Theoretische Informationstechnik, \\ Technische Universität München, Arcisstraße 21, 80333 München, Germany \\ $\dagger$ Fraunhofer Institute for Telecommunications, Heinrich-Hertz-Institute, \\ Einsteinufer 37, 10587 Berlin, Germany
}

\begin{abstract}
It is already known that the superposition property of wireless multiple-access channels can profitably be exploited for computing linear functions of the measurements in sensor networks. If appropriate pre- and post-processing functions are employed to operate on sensor readings and the superimposed signal received by a fusion center, respectively, then every function of the measurements is essentially computable by means of the channel at a single channel use, provided that pre- and postprocessing functions are not confined to be continuous. If the continuity property is required, then it has been recently shown that in general extra resources are necessary, thereby reducing the computation efficiency. In this paper we extend these results to the problem of computing vector-valued functions in clustered sensor networks (i.e., in networks of multiple-access channels) and show that if interference is appropriately harnessed, the component-functions can be computed much more efficiently than with standard approaches that avoid interference, even in the case of continuous pre- and post-processing functions.
\end{abstract}

\section{INTRODUCTION}

One of the major challenges in the wireless sensor network design is an efficient and universal computation of functions of the measurements at designated fusion centers [1]. Efficiency refers here to the ability of a network to effectively use wireless communication resources, while "universality" implies that transmit strategies are independent of the function to be computed: both properties lead to reduced energy consumptions and with it to extended network lifetimes.

Current wireless solutions to the computation problem avoid interference by using standard protocols such as time-division multiple access (TDMA) or carrier-sense multiple access (CSMA) to allow fusion centers the successive reconstruction of the entire raw sensor readings. This can be very inefficient since it was recently found that instead the natural superposition property of the wireless multiple-access channel (MAC) can profitably be exploited if fusion centers aim to compute ${ }^{1}$ linear functions [2], [3].

However, many functions of high practical relevance are nonlinear (e.g., maximum/minimum temperature in a measuring field), why the authors of [4] proposed a novel analog

\footnotetext{
${ }^{1}$ To avoid confusion with information-theoretic settings please note that whenever we write in this paper "computable", we exclusively mean analogcomputable (i.e., non-digital/uncoded).
}

computation scheme that employs appropriate pre-processing functions, operating on real-valued sensor readings prior to transmissions, and a post-processing function, operating on the real-valued superimposed signal received by a fusion center, to match the MAC to a nonlinear function of interest. Consequently, this abstraction of the wireless channel as an analog computer merges the processes of communication and computation, which are usually treated separately.

This approach is a recent example showing that analog systems become more important for wireless sensor networks, which is a development that is also confirmed by the fact that digital signal processing has in this context some fundamental limits [5]-[7].

\section{A. Preliminary Work}

Although some examples of nonlinear functions that are computable by means of a wireless MAC have been presented in [4], they are not universally computable since the corresponding pre-processing functions depend on the function to be computed at the fusion center. Above all, however, it remained unclear which functions are in general computable over the channel, why we investigated this question in [8] and found that the corresponding function space is equal to the space of nomographic functions (see Definition 1). If no additional restrictions on the pre-processing functions are imposed, then indeed every real multivariate function is nomographic and thus essentially computable via a MAC.

A surprising observation was also that the pre-processing functions can be chosen such that they are independent of the function the fusion center aims to evaluate at sensor readings, which results in universal computation schemes. Since the key advantage of this universality property is that one-way communication between the nodes and the fusion center suffices, feedback information about the function of interest is unnecessary so that the complexity of nodes can be further reduced.

\section{B. Contributions}

All these considerations assume that there is just a single fusion center attempting to compute a function of all 
measurements. In this paper, we extend the work of [8] to sensor networks that consist of multiple fusion centers, each of which aims at computing an arbitrary function of the sensor readings from a subset of nodes (i.e., clusters). This results in a problem of efficiently computing vector-valued functions over a network of multiple-access channels with a minimum amount of coordination.

In this context, we first show by a geometric interpretation of a famous theorem of Kolmogorov (see Theorem 2 and [8] $)^{2}$ that, in order to compute vector-valued functions over multiple-access channels with continuous pre- and postprocessing functions, a coordinated medium-access of clusters is not necessary provided that the fusion centers perform an additional simple processing step before applying their post-processing functions. The resulting computation scheme requires fewer wireless transmissions for function computation than standard TDMA-like protocols that coordinate the transmissions of clusters and nodes in time to avoid interference. Fortunately, unlike TDMA, the number of required transmissions is not proportional to the number of clusters. In addition, if no restrictions on pre- and post-processing functions are imposed, the efficiency can further be improved since additional properties such as continuity requires in general extra resources even though it can be beneficial in terms of implementations in practice.

Regardless of whether the pre-processing functions are continuous or not, the mentioned universality property is preserved in clustered sensor networks of arbitrary topology. As a consequence, simple one-way communication is sufficient in the entire network since there is no need to inform transmitting nodes about the functions to be computed at fusion centers.

Although the considerations are interesting in itself, the results of this paper can be also relevant for other sophisticated in-network processing such as gossip algorithms that intend to efficiently achieve a consensus with respect to a function based on local computations [10]-[12].

\section{Paper Organization}

The rest of the paper is organized as follows. In Section II we present some notational remarks, definitions and important theorems used throughout the paper, while Section III introduces the system model. Subsequently, in Section IV as the main section of the paper, we consider the problem of computing vector-valued functions in a network of multipleaccess channels and propose efficient computation schemes. Section V refers to some further useful properties that are inherent in the computation schemes from Section IV. Finally, Section VI concludes the paper.

\section{PReliminaries}

The $k$-times cartesian product $\mathbb{A} \times \cdots \times \mathbb{A}$ of a space $\mathbb{A}$ is written as $\mathbb{A}^{k}$. The natural and real numbers are denoted by $\mathbb{N}, \mathbb{R}=(-\infty, \infty)$, respectively, and $\mathbb{E}:=[0,1] \subset \mathbb{R}$ defines the closed unit interval. Let $\mathbb{A}^{k}$ be a compact metric space,

\footnotetext{
${ }^{2}$ The theorem disproves the conjecture stated by David Hilbert in his famous $13^{\text {th }}$ problem [9].
}

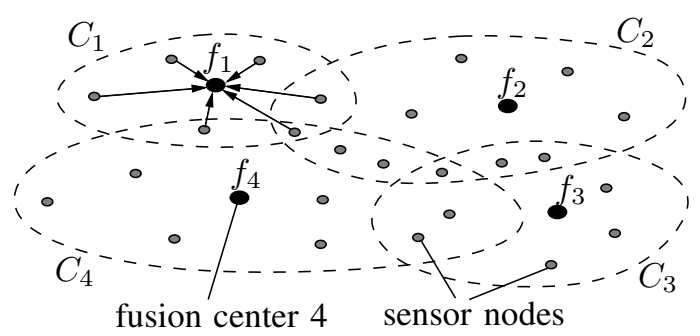

Fig. 1. A qualitative representation of a clustered wireless sensor network consisting of $N=25$ nodes and $K=4$ clusters for computing any functions $f_{1}, \ldots, f_{4}$ at fusion centers.

then $\mathcal{C}\left[\mathbb{A}^{k}\right]$ denotes with the infinity norm $\|\cdot\|_{\infty}$ the Banach space of real-valued continuous functions of $k \in \mathbb{N}$ variables, defined on $\mathbb{A}^{k}$. Furthermore, $\mathcal{F}\left[\mathbb{B}^{\ell}\right]$ denotes the space of any function $g: \mathbb{B}^{\ell} \subseteq \mathbb{R}^{\ell} \rightarrow \mathbb{R}, \ell \in \mathbb{N}$. Finally, $\mathcal{N}\left[\mathbb{A}^{k}\right]$ denotes the space of nomographic functions with domain $\mathbb{A}^{k}$ which are defined as follows.

Definition 1 (Nomographic Functions). Let $\mathbb{A}^{k}, k \geq 2$, be a metric space. Then, every $f \in \mathcal{F}\left[\mathbb{A}^{k}\right]$ for which a representation

$$
f\left(x_{1}, \ldots, x_{k}\right)=\psi\left(\sum_{i=1}^{k} \varphi_{i}\left(x_{i}\right)\right)
$$

exists, with $\psi \in \mathcal{F}[\mathbb{R}]$ and $\varphi_{i} \in \mathcal{F}[\mathbb{A}]$, for all $i=1, \ldots, k$, is called nomographic function.

Theorem 1 (Buck'79 [13]). Every $f \in \mathcal{F}\left[\mathbb{E}^{k}\right]$ is nomographic (i.e., $\mathcal{N}\left[\mathbb{E}^{k}\right]=\mathcal{F}\left[\mathbb{E}^{k}\right]$ ).

In contrast to $\mathcal{N}\left[\mathbb{A}^{k}\right], \mathcal{N}_{0}\left[\mathbb{A}^{k}\right]$ denotes in the following the space of nomographic functions with restrictions $\psi \in \mathcal{C}[\mathbb{R}]$ and $\varphi_{i} \in \mathcal{C}[\mathbb{A}], i=1, \ldots, k$. In this context, Theorem 1 is no longer valid such that the following important theorem will be useful for the considerations in this paper as well.

Theorem 2 (Kolmogorov'57 [14]). Every function $f \in \mathcal{C}\left[\mathbb{E}^{k}\right]$ can be represented in the form

$$
f\left(x_{1}, \ldots, x_{k}\right)=\sum_{j=0}^{2 k} g_{j}\left(x_{1}, \ldots, x_{k}\right),
$$

with $2 k+1$ nomographic functions $g_{j}\left(x_{1}, \ldots, x_{k}\right)=$ $\psi_{j}\left(\sum_{i=1}^{k} \varphi_{i j}\left(x_{i}\right)\right) \in \mathcal{N}_{0}\left[\mathbb{E}^{k}\right]$, where only the functions $\psi_{j}$ depend on $f$ but the $k(2 k+1)$ functions $\varphi_{i j}$ do not.

Theorem 3 (Sternfeld'85 [15]). To represent every $f \in \mathcal{C}\left[\mathbb{E}^{k}\right]$ in the form of (2), there are at least $2 k+1$ nomographic functions necessary (i.e., $2 k+1$ can not be reduced).

For more details, the reader is referred to [8].

\section{SySTEM MODEL}

Consider a wireless sensor network consisting of $N \in \mathbb{N}$ spatially distributed nodes that monitor the environment resulting in sensor readings $x_{n} \in \mathbb{E}, n=1, \ldots, N$. Assume that the network is organized into $K \in \mathbb{N}$ clusters, where the set of nodes belonging to cluster $k$ is denoted by $C_{k}$, 
$k=1, \ldots, K$. Each cluster, consisting of $\left|C_{k}\right|$ nodes, has a unique node designated as the fusion center and we assume that $C_{k} \bigcap C_{\ell} \neq \varnothing$, for all $k, \ell$ (see Fig. 1).

To describe the intra-cluster communication between nodes and corresponding fusion centers, we use the standard affine model of a wireless multiple-access channel such that the realvalued signal received by fusion center $k$ can be written as

$$
y_{k}=\sum_{n \in C_{k}} h_{k n} s_{k n}\left(x_{n}\right)+v_{k}, k=1, \ldots, K
$$

where $s_{k n}: \mathbb{E} \rightarrow \mathbb{R}$ denotes a transmit signal of node $n \in$ $C_{k}$ depending on sensed value $x_{n}, h_{k n} \in \mathbb{R}$ is a flat-fading coefficient between node $n$ and fusion center $k$ and $v_{k} \in \mathbb{R}$ is receiver noise, respectively. Ignoring in (3) the fading and the noise results then for $k=1, \ldots, K$ in ideal MACs

$$
\left(x_{k_{1}}, \ldots, x_{k_{\left|C_{k}\right|}}\right) \mapsto \sum_{n \in C_{k}} s_{k n}\left(x_{n}\right)
$$

that are connected by the common nodes. The mappings (4) highlight summation as the natural mathematical operation of wireless MACs (see Remark 2).

Remark 1. Note that (3) as well as (4) implies that only the nodes belonging to cluster $C_{k}$ are able to reach the $k^{\text {th }}$ fusion center, $k=1, \ldots, K$. This coincides with a scenario in which clusters are formed due to the connectivity radius of the $N$ spatially distributed nodes. In other words, there are $K$ independent fusion centers that aim at exploiting the public observations of all nodes to compute any function, but they are limited to node subsets only due to reachability constraints.

In [2], [3] it is shown that superpositions (4) caused by simultaneously transmitting nodes can profitably be exploited if fusion centers aim at computing linear functions of the measurements only. To enable the computation of nonlinear functions by means of the wireless MACs as well (cf. Definition 1), we employ appropriate pre- and post-processing functions defined as follows.

Definition 2 (Pre-Processing Functions). We define the real functions $\varphi_{k n}: \mathbb{E} \rightarrow \mathbb{R}$, operating on the sensor readings $x_{n}, n \in C_{k}, k=1, \ldots, K$ (i.e., $\varphi_{k n}\left(x_{n}\right)$ ), to be the preprocessing functions.

Definition 3 (Post-Processing Functions). Let $y_{k} \in \mathbb{R}$ be the output of the wireless MAC according to (3). Then, we define the univariate functions $\psi_{k}: \mathbb{R} \rightarrow \mathbb{R}, k=1, \ldots, K$, operating on $y_{k}$ (i.e., $\psi_{k}\left(y_{k}\right)$ ), to be the post-processing functions.

Remark 2. Although practical computation schemes suffer from limitations such as power constraints, fading, receiver noise, synchronization issues, we consider in this paper with $s_{k n} \equiv \varphi_{k n}$, for all $k, n$, computations over ideal MACs (4) only to focus on the core of the computation problem. The extension to realistic MACs (3) follows along similar lines such as in [16]. Furthermore, desired properties (e.g., universality) carry over to the practical setting so that the simplification does not impacts the generality of the results obtained in this paper.

\section{Analog Computation of Vector-Valued FUNCTIONS}

We view the clusters $C_{k}, k=1, \ldots, K$, as collections of distributed computation devices with the aim of efficiently determining desired functions $f_{k}\left(x_{k_{1}}, \ldots, x_{k_{\left|C_{k}\right|} \mid}\right)$, $k=1, \ldots, K$, of corresponding sensor readings at fusion centers, where a strategy is said to be more efficient if it needs less wireless resources and less coordination to compute function values with the same precision. Therefore, the task is to compute the vector-valued function $f: \mathbb{E}^{N} \rightarrow \mathbb{R}^{K}$,

$$
\boldsymbol{f}\left(x_{1}, \ldots, x_{N}\right):=\left(\begin{array}{c}
f_{1}\left(x_{1_{1}}, \ldots, x_{1_{\left|C_{1}\right|}}\right) \\
\vdots \\
f_{K}\left(x_{K_{1}}, \ldots, x_{K_{\left|C_{K}\right|}}\right)
\end{array}\right)
$$

by means of the network of ideal MACs (4). In doing so we consider in Section IV-A the case where only continuous preand post-processing functions are allowed while in Section IV-B no restrictions on pre- and post-processing functions are imposed.

\section{A. Continuous Pre- and Post-Processing Functions}

1) Geometry and Scheme: If continuous pre- and postprocessing functions are used, Theorem 2 in conjunction with Theorem 3 states that, to compute every continuous function of $N$ sensor readings, a sum over $2 N+1$ nomographic functions from $\mathcal{N}_{0}\left[\mathbb{E}^{N}\right]$ is required [8] (cf. Remark 5). This can be achieved if all nodes carry out $2 N+1$ successive and simultaneous transmissions followed by an additional postprocessing step at fusion centers described below. According to Theorem 2, however, one could adopt that in each cluster, say cluster $k, 2\left|C_{k}\right|+1$ successive transmissions are sufficient to compute every continuous function of $\left|C_{k}\right|$ variables. Because of the couplings between clusters due to common nodes this would, however, require a large amount of coordination ${ }^{3}$ and a constant adaptation of the pre-processing on the common sensor nodes, which is explained in the following.

Consider a single cluster containing all $N$ nodes of the network that aims to compute an arbitrary function $f \in \mathcal{C}\left[\mathbb{E}^{N}\right]$ (i.e., all nodes are able to reach a single fusion center). Then, Theorem 2 implies that the corresponding $2 N+1$ successive transmissions of all nodes generate a continuous and bijective correspondence $\left(x_{1}, \ldots, x_{N}\right) \mapsto\left(y_{0}, \ldots, y_{2 N}\right)$,

$$
\left(\begin{array}{c}
y_{0} \\
\vdots \\
y_{2 N}
\end{array}\right):=\left(\begin{array}{c}
\varphi_{10}\left(x_{1}\right)+\cdots+\varphi_{N 0}\left(x_{N}\right) \\
\vdots \\
\varphi_{1,2 N}\left(x_{1}\right)+\cdots+\varphi_{N, 2 N}\left(x_{N}\right)
\end{array}\right) \in \Gamma,
$$

between sensor readings and ideal MAC output-signals $y_{m}$, $m=0, \ldots, 2 N$, with $\Gamma$ a compact subset of the Euclidean space $\mathbb{R}^{2 N+1}$. In other words, (6) describes a homeomorphism between $\mathbb{E}^{N}$ and $\Gamma$ such that $\mathbb{E}^{N}$ is continuously embedded into $\mathbb{R}^{2 N+1}$, for which reason (6) is nothing else than a bijective correspondence between all continuous

\footnotetext{
${ }^{3}$ This in turn requires two-way communication between nodes and fusion centers which increases the complexity of nodes.
} 
functions $f\left(x_{1}, \ldots, x_{N}\right)$ on $\mathbb{E}^{N}$ and all continuous functions $F\left(y_{0}, \ldots, y_{2 N}\right)$ on $\Gamma$.

Would now only a subset of the nodes participate in the $2 N+1$ transmissions (i.e., some summands on the right hand side in (6) are missing), then the image $\left(y_{0}, \ldots, y_{2 N}\right)$ is not necessarily in $\Gamma$ which in turn implies that not every $F \in \mathcal{C}[\Gamma]$ is computable. This is exactly what happens if the network is organized into overlapping clusters, where each cluster independently aims to compute a component-function of $f$ that depends only on a subset of the nodes.

Fortunately, this fact can be easily and independently solved on each fusion center. For this purpose let us exploit a result from [17] which states that the $N(2 N+1)$ pre-processing functions on the right hand side of (6) can be chosen to be

$$
\varphi_{n m}\left(x_{n}\right)=\alpha_{n} \varphi\left(x_{n}+m \beta\right),
$$

with $\varphi$ being a well-defined, continuous and monotone increasing function, and $\left\{\alpha_{n}\right\}_{n=1}^{N}, \beta$ appropriate nonnegative real constants. Both $\varphi$ and the constants are independent of $f$. Then, the $2 N+1$ successively received ideal MAC outputsignals at the $K$ fusion centers can be summarized in the vectors

$$
\boldsymbol{y}_{k}=\left(\begin{array}{c}
y_{k 0} \\
\vdots \\
y_{k, 2 N}
\end{array}\right)=\left(\begin{array}{c}
\sum_{n \in C_{k}} \alpha_{n} \varphi\left(x_{n}\right) \\
\vdots \\
\sum_{n \in C_{k}} \alpha_{n} \varphi\left(x_{n}+2 N \beta\right)
\end{array}\right),
$$

$k=1, \ldots, K$, which are in general not points in $\Gamma$. However, if we consider the shifted versions

$$
\boldsymbol{z}_{k}:=\boldsymbol{y}_{k}+\gamma_{k}
$$

with

$$
\gamma_{k}=\left(\begin{array}{c}
\gamma_{k 0} \\
\vdots \\
\gamma_{k, 2 N}
\end{array}\right):=\left(\begin{array}{c}
\sum_{n \notin C_{k}} \alpha_{n} \varphi(0) \\
\sum_{n \notin C_{k}} \alpha_{n} \varphi(\beta) \\
\vdots \\
\sum_{n \notin C_{k}} \alpha_{n} \varphi(2 N \beta)
\end{array}\right),
$$

then unlike $\boldsymbol{y}_{k}, \boldsymbol{z}_{k}=\left(z_{k 0}, \ldots, z_{k, 2 N}\right)$ is a member of $\Gamma$, for all $k=1, \ldots, K$. It is important to emphasize that $\gamma_{k} \in$ $\mathbb{R}^{2 N+1}$ is a constant, and therefore does not depend on the sensor readings.

Now, provided that each fusion center knows $N$, we conclude from (9) a simple post-processing at fusion centers that enables the efficient computation of every vector-valued function consisting of continuous component-functions.

After receiving in the $m^{\text {th }}$ time-slot, $m=0, \ldots, 2 N$, the ideal MAC output-signal $y_{\mathrm{km}}$ and after adding the correction term $\gamma_{k m}$, fusion center $k, k=1, \ldots, K$, applies the corresponding post-processing function to $z_{k m}$

$$
\psi_{k m}\left(z_{k m}\right)=\psi_{k m}\left(\sum_{n \in C_{k}} \alpha_{n} \varphi\left(x_{n}+m \beta\right)+\gamma_{k m}\right),
$$

and stores this intermediate result in a memory. Finally, if all $2 N+1$ ideal MAC output-signals are received and postprocessed, the fusion centers compute the desired componentfunctions by summing up the respective memory content to

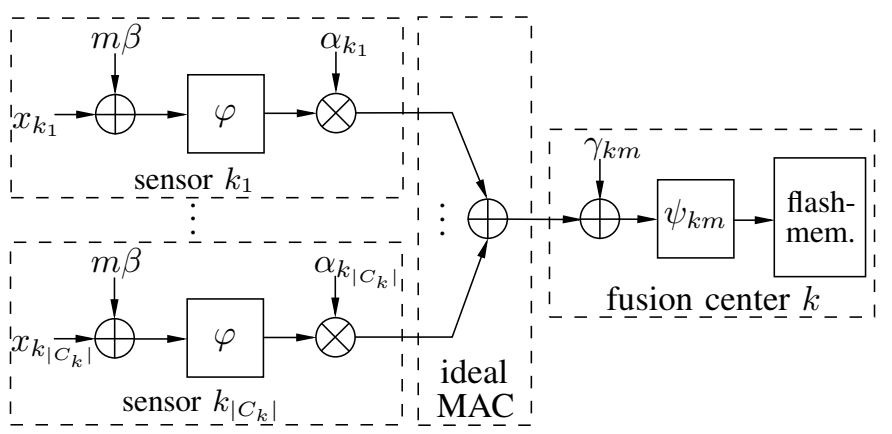

Fig. 2. Block diagram for computations in cluster $k, k=1, \ldots, K$, at time slot $m, m=0, \ldots, 2 N$, consisting of $\left|C_{k}\right|$ transmitting sensor nodes and the fusion center. After $2 N+1$ receptions, the fusion center has to sum up the flash memory content which results immediately in the desired function value $f_{k}\left(x_{k_{1}}, \ldots, x_{k_{\left|C_{k}\right|}}\right)$.

obtain

$$
\boldsymbol{f}\left(x_{1}, \ldots, x_{N}\right)=\left(\begin{array}{c}
\sum_{m=0}^{2 N} \psi_{1 m}\left(z_{1 m}\right) \\
\vdots \\
\sum_{m=0}^{2 N} \psi_{K m}\left(z_{K m}\right)
\end{array}\right) .
$$

A corresponding block diagram for a particular time slot $m$, $m=0, \ldots, 2 N$, is depicted in Fig. 2 .

Remark 3. Since the pre-processing functions are independent of the components of $f$, the fusion centers determine by appropriately choosing the $K(2 N+1)$ post-processing functions $\left\{\psi_{k m}\right\}$, which continuous functions $f_{1}, \ldots, f_{K}$ are to be computed. Moreover, the constructive proof of Theorem 2 in [17] provides an algorithm that can be used to determine the universal pre-processing function $\varphi$.

Remark 4. The constants $\alpha_{n}$ need not to be different for all $N$ nodes in the network such that they can be reused in different clusters without any kind of arrangement between them.

2) Performance: In the following we highlight the advantages of the computation approach depicted in Fig. 2 in a network of $K$ clusters over standard TDMA approaches. To this end, let us first consider the case mentioned at the beginning of Section IV-A1, where the clusters use only $2\left|C_{k}\right|+1$, $k=1, \ldots, K$, successive transmissions instead of $2 N+$ 1. Then, there exist homeomorphisms $\left(x_{k_{1}}, \ldots, x_{k_{\left|C_{k}\right|}}\right) \mapsto$ $\left(y_{k 0}, \ldots, y_{k, 2\left|C_{k}\right|}\right)$ between $\mathbb{E}^{\left|C_{k}\right|}$ and $\Gamma_{k} \subset \mathbb{R}^{2\left|C_{k}\right|+1}$ that enable each cluster to compute every $f_{k} \in \mathcal{C}\left[\mathbb{E}^{\left|C_{k}\right|}\right]$ because of the existence of representations

$$
f_{k}\left(x_{k_{1}}, \ldots, x_{k_{\left|C_{k}\right|}}\right)=\sum_{m=0}^{2\left|C_{k}\right|} \psi_{k m}\left(\sum_{n \in C_{k}} \varphi_{n m}^{(k)}\left(x_{n}\right)\right),
$$

$k=1, \ldots, K$. Since the compact sets $\Gamma_{k}$ will unfortunately differ in general, the pre-processing functions in (13) depend on $k$ such that the common nodes between clusters have to adapt their pre-processing functions in dependency of the fusion center to which they transmit next. This in turn requires the coordinated activation of clusters as indicated in Fig. 


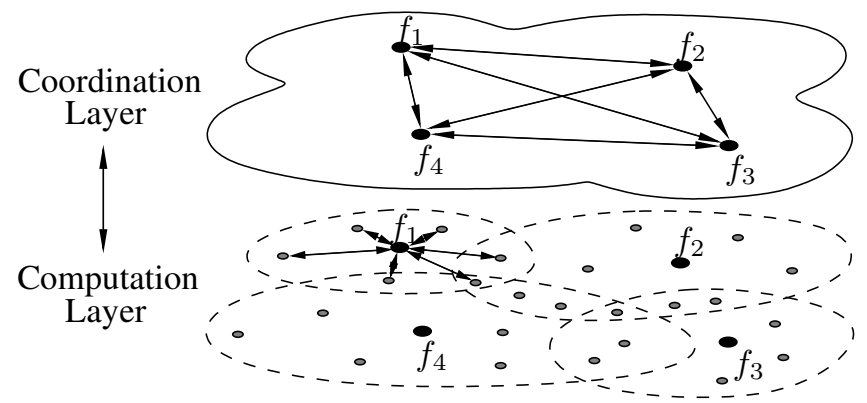

Fig. 3. The clustered sensor network example from Fig. 1 with an additional coordination layer that coordinates the medium-access of clusters such as in a standard TDMA approach. This requires bidirectional wireless communication links between the coordination layer and the computation layer as well as between the nodes and the fusion centers in each cluster (represented by arrows with two peaks).

3. But to guarantee an appropriate coordination, two-way communication between nodes and fusion centers has to be implemented. In contrast, the proposed computation method from Section IV-A1, depicted in Fig. 2, do not requires any global coordination since all clusters can transmit simultaneously such that one-way communication suffices. Furthermore, due to the universality property of pre-processing functions, cluster topologies are allowed to change without to reconfigure transmitting nodes.

If a standard TDMA protocol is used to compute vectorvalued functions in a clustered wireless sensor network, besides the orthogonalized medium-access of the nodes in each cluster, clusters themselves have to be appropriately separated in time (see Fig. 3). Unlike the $2 N+1$ transmissions, a standard TDMA would induce $K L \geq K \max _{k}\left|C_{k}\right|$ separated transmissions to convey the entire raw sensor readings interference-free to the $K$ fusion centers, which subsequently compute the component-functions $\left\{f_{k}\right\}_{k=1}^{K}$. In contrast to $K L$, it is obvious that $2 N+1$ does not scales with the number of clusters such that huge performance gains are possible.

Remark 5. We conclude from Theorem 3 that unfortunately the number $2 N+1$ of transmissions can not be reduced since otherwise there exists an $f \in \mathcal{C}\left[\mathbb{E}^{N}\right]$ that is not representable in the form (2).

\section{B. Arbitrary Pre- and Post-Processing Functions}

If no restrictions on pre- and post-processing functions are imposed, the situation is much less complicated than in the last subsection since Theorem 1 states that every function of $\ell \geq 2$ variables is nomographic according to $\mathcal{N}\left[\mathbb{E}^{\ell}\right]$. This means that already a single simultaneous transmission of the nodes in each cluster is sufficient to compute every $f \in \mathcal{F}\left[\mathbb{E}^{\left|C_{1}\right|}\right] \times \cdots \times$ $\mathcal{F}\left[\mathbb{E}^{\left|C_{K}\right|}\right]$. Consider therefore the ideal MAC output-signals

$$
y_{k}=g_{k}\left(x_{k_{1}}, \ldots, x_{k_{\left|C_{k}\right|}}\right)=\sum_{n \in C_{k}} \varphi_{n}\left(x_{n}\right)
$$

received by the fusion centers, which are mappings $g_{k}$ : $\mathbb{E}^{\left|C_{k}\right|} \rightarrow \Lambda_{k} \subset \mathbb{R}, k=1, \ldots, K$. Then, the post-processing at fusion centers consists merely in the application of appropriate post-processing functions such that every vector-valued function (5) can be represented as

$$
\boldsymbol{f}\left(x_{1}, \ldots, x_{N}\right)=\left(\begin{array}{c}
\psi_{1}\left(y_{1}\right) \\
\vdots \\
\psi_{K}\left(y_{K}\right)
\end{array}\right) \text {. }
$$

But what is the difference to the previous case where continuous pre- and post-processing functions are desired?

We know from [18, Lemma 1] that a necessary and sufficient condition to compute every vector-valued function (15) is that the functions $\left\{g_{k}\right\}_{k=1}^{K}$, such as defined in (14), are bijective. In other words, the pre-processing functions $\left\{\varphi_{n}\right\}_{n=1}^{N}$ have to be chosen such that for all sensor readings $\left(x_{1}^{(1)}, \ldots, x_{N}^{(1)}\right) \in \mathbb{E}^{N},\left(x_{1}^{(2)}, \ldots, x_{N}^{(2)}\right) \in \mathbb{E}^{N}$, with $\left(x_{1}^{(1)}, \ldots, x_{N}^{(1)}\right) \neq\left(x_{1}^{(2)}, \ldots, x_{N}^{(2)}\right)$, and all $k=1, \ldots, K$, always $\sum_{n \in C_{k}} \varphi_{n}\left(x_{n}^{(1)}\right) \neq \sum_{n \in C_{k}} \varphi_{n}\left(x_{n}^{(2)}\right)$ holds. This can never be achieved for every $f$ with continuous pre- and postprocessing functions [18], why it was necessary in Section IV-A to appropriately embed $\mathbb{E}^{N}$ into a higher dimensional space, resulting in a bijection between function spaces $\mathcal{C}\left[\mathbb{E}^{N}\right]$ and $\mathcal{C}[\Gamma]$ instead. If pre- and post-processing functions are allowed to be discontinuous, however, such an embedding is superfluous.

Remark 6. Note that similar to the continuous case (see Remark 3), the pre-processing functions in (14) can be chosen to be independent of the component-functions $f_{k}, k=1, \ldots, K$, the fusion centers intend to compute. Thus, they decide by an appropriate choice of the post-processing functions $\left\{\psi_{k}\right\}_{k=1}^{K}$ which component-functions are computed. This implies once more that one-way communication between nodes and fusion centers is sufficient since coordination is not needed.

\section{Further Properties}

In this section we refer to some further useful properties that are implied by the approaches proposed in Section IV.

Due to the universality of the computation approaches from Sections IV-A and IV-B, they guarantee a kind of "embedded security". Assume therefore, that an eavesdropper tries to identify which component-function is computed at a particular fusion center. Then, this attempt is hopeless even if the eavesdropper knows all transmit strategies (i.e., preprocessing functions), since the pre-processing functions are independent of the function the fusion center wants to compute. Hence, eavesdropping superimposed transmit-signals is useless as a result of the fact that each fusion center determines the component-function by employing corresponding postprocessing functions.

Another useful property is the robustness of the proposed computation schemes against variations in the topology of clusters. In other words, the universality property is preserved if nodes drop out of the network (due to for instance failures or battery depletion). If no restrictions on pre-processing functions are imposed, this follows immediately from [18, Theorem 1]. In the case of continuous pre- and post-processing 
functions, the fusion centers only need to adjust the shift (10) by appropriately adding further constants that correspond to the dropped out nodes.

\section{CONCLUSION}

In the present paper we investigated the problem of analog computing vector-valued functions in clustered wireless sensor networks, where nodes were allowed to transmit simultaneously to exploit the natural superposition property of multipleaccess channels. By employing appropriate pre- and postprocessing functions operating on real sensor readings and on the superimposed signals received by designated fusion centers, respectively, essentially an unlimited number of linear and nonlinear functions is universally computable over the channel. Universality means in this context that the preprocessing functions are independent of the functions to be computed at fusion centers such that they do not need to be updated if desired functions change. In other words, universality implies that coordination is not needed and thus one-way communication between nodes and fusion centers suffices.

If no restrictions on pre- and post-processing functions are imposed, we have shown that in fact every function on each fusion center can be efficiently and universally computed, where the number of required transmissions/time-slots is of the order $\mathcal{O}(1)$, while a standard TDMA approach requires $\mathcal{O}(K L)$ transmissions. This number scales with the number $K$ of clusters and with the number $L$ of nodes belonging to the largest cluster such that huge performance gains are possible if computations are performed over the channel.

Since for practical implementations continuous pre- and post-processing functions are preferable, a corresponding restriction generates the need for an additional processing step at fusion centers to ensure the computability of every continuous multivariate function. Moreover, the continuity of pre- and post-processing functions requires additional wireless resources. In this context, we have shown that the number of required transmissions to compute an arbitrary vectorvalued function with continuous component-functions is of the order $\mathcal{O}(2 N+1)$, which offers the potential of significant performance gains in comparison to a standard TDMA, since the number of transmissions is proportional to the number $N$ of nodes but not proportional to the number of clusters $K$.

The work in this paper demonstrates that analog systems are well suited to efficiently solve arbitrary computation problems in wireless sensor networks. Indeed, it was recently even shown in [6] that with ordinary sampling, purely analog linear systems are not always stable representable in discrete time domain. Moreover, sampling is usually followed by quantization, which generates additional instabilities that are not always controllable by oversampling [5], [7]. Thus, digital signal processing has some fundamental limits and analog systems are gaining attention in the sensor network community.

Remark 7. Finally, we point out that we considered in this paper sensor readings which are drawn from the unit interval $\mathbb{E}$. This, however, is in no way a loss in generality since
Theorem 2 were for example in [19] extended to continuous functions that are defined over arbitrary compact metric spaces of covering dimension $k \in \mathbb{N}$. Similarly, Theorem 3 holds for arbitrary compact metric spaces [15].

\section{ACKNOWLEDGMENT}

The work of M. Goldenbaum and S. Stańczak was supported in part by the German Research Foundation (DFG) under grant STA $864 / 3-1$ and the work of $\mathrm{H}$. Boche by the DFG under grant BO 1734/22-1, respectively.

\section{REFERENCES}

[1] A. Giridhar and P. R. Kumar, "Toward a theory of in-network computation in wireless sensor networks," IEEE Commun. Mag., vol. 44, no. 4, pp. 98-107, Apr. 2006.

[2] M. Gastpar and M. Vetterli, "Source-channel communication in sensor networks," in Proc. 2nd International Workshop on Information Processing in Sensor Networks (IPSN), Palo Alto, USA, Apr. 2003, pp $162-177$.

[3] S. Stańczak, M. Wiczanowski, and H. Boche, "Distributed utility-based power control: Objectives and algorithms," IEEE Trans. Signal Process., vol. 55, no. 10 , pp. 5058-5068, Oct. 2007.

[4] M. Goldenbaum, S. Stańczak, and M. Kaliszan, "On function computation via wireless sensor multiple-access channels," in Proc. IEEE Wireless Communications \& Networking Conference (WCNC), Budapest, Hungary, Apr. 2009.

[5] H. Boche and U. J. Mönich, "Behavior of the quantization operator for bandlimited, nonoversampled signals," IEEE Trans. Inf. Theory, vol. 56, no. 5, pp. 2433-2440, May 2010.

[6] - "Sampling of deterministic signals and systems," IEEE Trans. Signal Process., vol. 59, no. 5, pp. 2101-2111, May 2011.

[7] — , "Unboundedness of thresholding and quantization for bandlimited signals," Signal Processing, 2012, submitted for publication.

[8] M. Goldenbaum, H. Boche, and S. Stańczak, "Analyzing the space of functions analog-computable via wireless multiple-access channels," in Proc. 8th International Symposium on Wireless Communication Systems (ISWCS), Aachen, Germany, Nov. 2011, pp. 779-783.

[9] D. Hilbert, "Mathematical problems," Bull. Amer. Math. Soc., vol. 8, no. 10, pp. 437-479, 1902, engl. translation.

[10] R. Olfati-Saber, J. A. Fax, and R. M. Murray, "Consensus and cooperation in networked multi-agent systems," Proc. IEEE, vol. 95, no. 1, pp. 215-233, Jan. 2007.

[11] D. Mosk-Aoyama and D. Shah, "Fast distributed algorithms for computing separable functions," IEEE Trans. Inf. Theory, vol. 54, no. 7, pp. 2997-3007, Jul. 2008.

[12] A. G. Dimakis, S. Kar, J. M. F. Moura, M. G. Rabbat, and A. Scaglione, "Gossip algorithms for distributed signal processing," Proc. IEEE, vol. 98, no. 11, pp. 1847-1864, Nov. 2010.

[13] R. C. Buck, "Approximate complexity and functional representation," $J$. Math. Anal. Appl., vol. 70, pp. 280-298, 1979.

[14] A. N. Kolmogorov, "On the representation of continuous functions of several variables by superposition of continuous functions of one variable and addition," Dokl. Akad. Nauk SSSR, no. 114, pp. 953-956, 1957, engl. translation.

[15] Y. Sternfeld, "Dimension, superposition of functions and separation of points, in compact metric spaces," Israel J. Math., vol. 50, pp. 13-52, 1985.

[16] M. Goldenbaum and S. Stańczak, "Computing functions via SIMO multiple-access channels: How much channel knowledge is needed?" in Proc. IEEE International Conference on Acoustics, Speech and Signal Processing (ICASSP), Dallas, USA, Mar. 2010, pp. 3394-3397.

[17] J. Braun and M. Griebel, "On a constructive proof of Kolmogorov's superposition theorem," Constr. Approx., vol. 30, no. 3, pp. 653-675, Dec. 2009.

[18] M. Goldenbaum, H. Boche, and S. Stańczak, "Analog computation via wireless multiple-access channels: Universality and robustness," in Proc. IEEE International Conference on Acoustics, Speech and Signal Processing (ICASSP), Kyoto, Japan, Mar. 2012.

[19] P. A. Ostrand, "Dimension of metric spaces and Hilbert's problem 13," Bull. Amer. Math. Soc., vol. 71, no. 4, pp. 619-622, Jul. 1965. 